2nd International Conference on Ultrafine Grained \&

Nanostructured Materials (UFGNSM)

International Journal of Modern Physics: Conference Series

Vol. 5 (2012) 168-176

(C) World Scientific Publishing Company

DOI: 10.1142/S2010194512001985

\title{
Platinum nano particles dispersed in alumina
}

\author{
Abedeh Gholidoust ${ }^{1}$ \\ Department of Chemical engineering, \\ Faculty of engineering, Amirkabir University of technology, Tehran, Iran \\ gholidoost@aut.ac.ir \\ Abbas Naderifar \\ Department of Chemical engineering, \\ Faculty of engineering, Amirkabir University of technology, P.O.Box 15875-4413, Tehran, Iran \\ Naderifar@aut.ac.ir \\ Mohammad Rahmani \\ Department of Chemical engineering, \\ Faculty of engineering, Amirkabir University of technology, P.O.Box 15875-4413, Tehran, Iran \\ M.rahmani@aut.ac.ir \\ Saeed Sahebdelfar \\ Catalysis Research group, Tehran center, Petrochemical Research \&Technology Company NPC, \\ P.O.Box 14965-115, Tehran, Iran \\ s.sahebdel@npc-rt.ir
}

\begin{abstract}
We report the propane dehydrogenation behavior of catalysts prepared using wet impregnation method that immobilize Pt nano cluster on the alumina surface. The immobilization of the metal particles and their nano size dimensions were demonstrated by transmission electron microscopy. Selectivity to propylene for these catalysts is comparable to those obtained over industrial $\mathrm{Pt}$ catalysts, yet the resistance to deactivation by carbon poisoning is much greater for our catalysts. The deactivation behavior is more typical of traditionally prepared PtSn catalysts on $\gamma$-alumina than of catalysts supported on $\theta$-alumina.
\end{abstract}

Keywords: Platinum, nano, catalyst, propane dehydrogenation

\footnotetext{
${ }^{1}$ Department of chemistry, Faculty of engineering, UCP, ENSTA, 32 boulevard Victor, 75015 Paris
} 


\section{Introduction}

Noble metals such as platinum, palladium and rhodium have been used as an active phase in various hydrogenation and dehydrogenation processes in the petrochemical industry. Most of the noble metal catalysts have been prepared by impregnating the noble metals on highly stable inorganic supports with appropriate interaction such as alumina, silica, and activated carbon $^{1,2}$. The high dispersion of the noble metals on the supports is not only crucial in preparing highly active catalysts with a large number of exposed metal atoms, but it also reduces the loading levels of noble metals, thereby reducing the production costs. The high dispersion of noble metals on catalyst supports, therefore, makes it possible to prepare lowpriced noble metal catalysts having a high-catalytic activity with sufficient thermal and mechanical stabilities.

$\gamma$-Alumina has been commonly used as a support for noble metals because its moderate interaction with them results in their high dispersion and stability ${ }^{3}$. Its large surface area and high thermal stability also contributes to its high performance as a catalyst support. The weak acidity of $\gamma$-alumina, however, causes the formation of side products in organic reactions that takes place on its acid sites, thereby lowering the yields of the desired products. The deposition of carbon on the acid sites also reduces the service life of the noble metal catalysts supported on $\gamma$-alumina. Moreover, the poor accessibility of $\gamma$-alumina, due to the lack of hydroxyl groups on its surface, limits the shapes of the catalysts that can be prepared.

Another promising catalyst support for noble metals is $\theta$-Alumina. The inertness of $\theta$ Alumina suppresses the formation of side products and coke deposition. It is suggested that the strong interaction, however, influences the chemical state of noble metals and may cause considerable changes in their activities in catalytic reactions.

The preparation of a catalyst operating at high activity and selectivity wrestles with several difficulties.

The mechanical and thermal stability of a catalyst is strongly influenced by the nature of the precursors and the preparation methods applied for manufacturing the catalytic matter or on the pretreatment of catalyst preceding the catalytic runs ${ }^{4,5}$.

The general procedures for preparation of supported metal catalysts can be classified into three groups. The first includes the impregnation of the support by the appropriate solution of metal salt followed by the decomposition of this salt and the reduction of the metal ions to form metal nanoparticles. In the second procedure generally used for support matter of ion exchange properties, such as zeolites, etc., the exchange capacity is utilized to introduce the metal ion. The third procedure is the vapor deposition in which the volatile metal compound is deposited on the support.

In this study, we modified the surface character of $\gamma$-Alumina support by calcination to remove acid sites. The platinum precursor of cationic type was impregnated on $\theta$-Alumina support. Platinum catalysts impregnated on $\theta-\mathrm{Al}_{2} \mathrm{O}_{3}$ supports were obtained by subsequent 
drying and calcinations followed by reduction. We implemented XRD technique to confirm the $\theta$-phase construction. TEM analysis has also been accomplished to investigate the platinum particle size and its dispersion on both supports. In addition, the catalytic activities of platinum catalysts prepared were studied in the dehydrogenation of propane to verify the effect of the interaction between platinum and alumina on the catalytic performance of platinum.

\section{Experimental}

\subsection{Preparation of catalysts}

$\theta$-Alumina was prepared from $\gamma$-Alumina (Axens $\mathrm{AL}$ spherical $\gamma-\mathrm{Al}_{2} \mathrm{O}_{3}$ ) by calcination at $1050^{\circ} \mathrm{C}$ for $8 \mathrm{~h}$. XRD measurements proved complete conversion of $\gamma-\mathrm{Al}_{2} \mathrm{O}_{3}$ to $\theta-\mathrm{Al}_{2} \mathrm{O}_{3}$. Hexachloroplatinic acid solution was prepared by adding di-hydrogen hexachloroplatinate (IV) (Merck-807340) to deionized water. A rotary evaporator was used to contact the pellets with acid solution for desired time. Bath temperature was $25^{\circ} \mathrm{C}$, and it implemented under atmospheric pressure.

The solution was removed by using vacuum apparatus. Then they were dried at $120^{\circ} \mathrm{C}$ for $18 \mathrm{~h}$ to immobilize chloroplatinate ions. The pellets were calcinated under a stream of air from 25 to $500^{\circ} \mathrm{C}$ over a period of two and half an hour. For the second step we impregnated platinum catalysts with tin chloride II solution using $\mathrm{SnCl}_{2}$ (97\%, Johnson-7772-99-8). Chloridric acid (37\% wt., Merck-100314) was used as an appropriate solvent for tin chloride. The subsequent drying and calcinations steps are as the same as before.

Finally, the platinum catalysts supported on the alumina were obtained by reduction at 400 ${ }^{\circ} \mathrm{C}$ in a reducing gas flow $\left(\mathrm{H}_{2} / \mathrm{N}_{2}=2 / 1\right.$ as mole $)$. As a reference catalyst, the platinum catalyst impregnated on the $\gamma$-alumina was also prepared.

\subsection{Characterization}

The Brunauer-Emmett-Teller (BET) surface area, pore volume, and pore diameter of catalysts were measured by a $\mathrm{N}_{2}$ adsorption-desorption isotherm at liquid nitrogen temperature (77 K) using NOVA 2000 Series instrument (Quantachrome, Boynton Beach, FL). A PW-1800 Philips X-ray diffract meter with monochrome $\mathrm{Cu} \mathrm{K} \alpha$ radiation $(\lambda=1.5406$ $\AA$ ) was used for X-ray measurement. The conversion of $\gamma-\mathrm{Al}_{2} \mathrm{O}_{3}$ to $\theta-\mathrm{Al}_{2} \mathrm{O}_{3}$ was evaluated from the full width at half-maximum of the $\mathrm{CuO}$ (111) X-ray diffraction (XRD) peaks.

Table 1 BET results

\begin{tabular}{ccc}
\hline Sample & Specific Surface area $\left(\mathrm{m}^{2} / \mathrm{g}\right)$ & Pore volume $\left(\mathrm{cm}^{3} / \mathrm{g}\right)$ \\
\hline$\gamma-\mathrm{Al}_{2} \mathrm{O}_{3}$ & 132 & 1.05 \\
$\theta-\mathrm{Al}_{2} \mathrm{O}_{3}$ & 70.2837 & 0.512 \\
\hline
\end{tabular}


The actual metallic loadings of prepared catalysts were determined by inductively coupled plasma (ICP), using a VISTA-PRO. The transmission electron micrographs of the platinum catalysts were obtained on a Jeol JEM-2000FXII electron microscope operating at $200 \mathrm{kV}$. The amount of coke adsorbed on catalysts was measured using thermo-gravimetric analyser (TGA) apparatus. The catalysts were heated from 40 to $1000{ }^{\circ} \mathrm{C}$, and then the weight loss of the catalysts was measured which is the net coke formed on the catalyst.

\section{3 catalytic reaction}

Dehydrogenation of propane on the platinum catalysts was carried out at the space velocity (WHSV) of $2.2 \mathrm{~h}^{-1}$, and the temperature was $620^{\circ} \mathrm{C}$, under 0.86 bar pressure.

The reactor temperature was increased with a ramping rate of $5{ }^{\circ} \mathrm{C} / \mathrm{min}$ from ambient temperature to $620{ }^{\circ} \mathrm{C}$. Reactor temperature is measured by three thermocouples externally attached to the top, middle, and bottom of the reactor, and one thermocouple located inside the reactor at the bottom of the catalyst bed.

One gram sample of the platinum catalysts was charged at the center of a quartz tube reactor (10 mm ID). The reactant was composed of propane (99.99\%) of $18.7 \mathrm{ml}$ and hydrogen (99.99\%) of $11.2 \mathrm{ml}\left(\mathrm{H}_{2} / \mathrm{HC}=0.6\right)$. In a typical reaction sequence, the catalyst is heated to $400{ }^{\circ} \mathrm{C}$ in flowing nitrogen, where upon nitrogen flow is stopped and reactant flow begun.

The composition of the effluent gas phase was measured by an online gas chromatograph (Agilent 6890) equipped with a flame ionization detector. Thermal conductivity detector was used to determine the hydrogen amount.

\section{Results}

We prepared three catalysts supported on both $\gamma$-alumina and $\theta$-alumina. The compositions of the platinum catalysts prepared are listed in Table 2 .

Table 2 catalyst composition (ICP mass spectroscopy)

\begin{tabular}{llll}
\hline Catalyst & \multicolumn{2}{l}{ Composition (wt\%) } & Pt Adsorption yield (\%) \\
\cline { 2 - 3 } & $\mathrm{Pt}$ & $\mathrm{Sn}$ & \\
1. Pt-Sn $/ \gamma-\mathrm{Al}_{2} \mathrm{O}_{3}$ & 0.5 & 0.25 & 97.23 \\
2.Pt-Sn $/ \theta-\mathrm{Al}_{2} \mathrm{O}_{3}$ & 0.5 & 0.25 & 96.17 \\
3.Pt-Sn $/ \boldsymbol{\theta}-\mathrm{Al}_{2} \mathrm{O}_{3}$ & 0.3 & 0.25 & 95.74 \\
\hline
\end{tabular}

The dispersive states of theta and gamma alumina induced different XRD patterns as shown in Fig. 1. The sharp peaks at 45.474 and $67.137^{\circ}$ attributed to gamma phase. The sharp peaks at 32.76 and $67.42^{\circ}$ attributed to theta phase. 

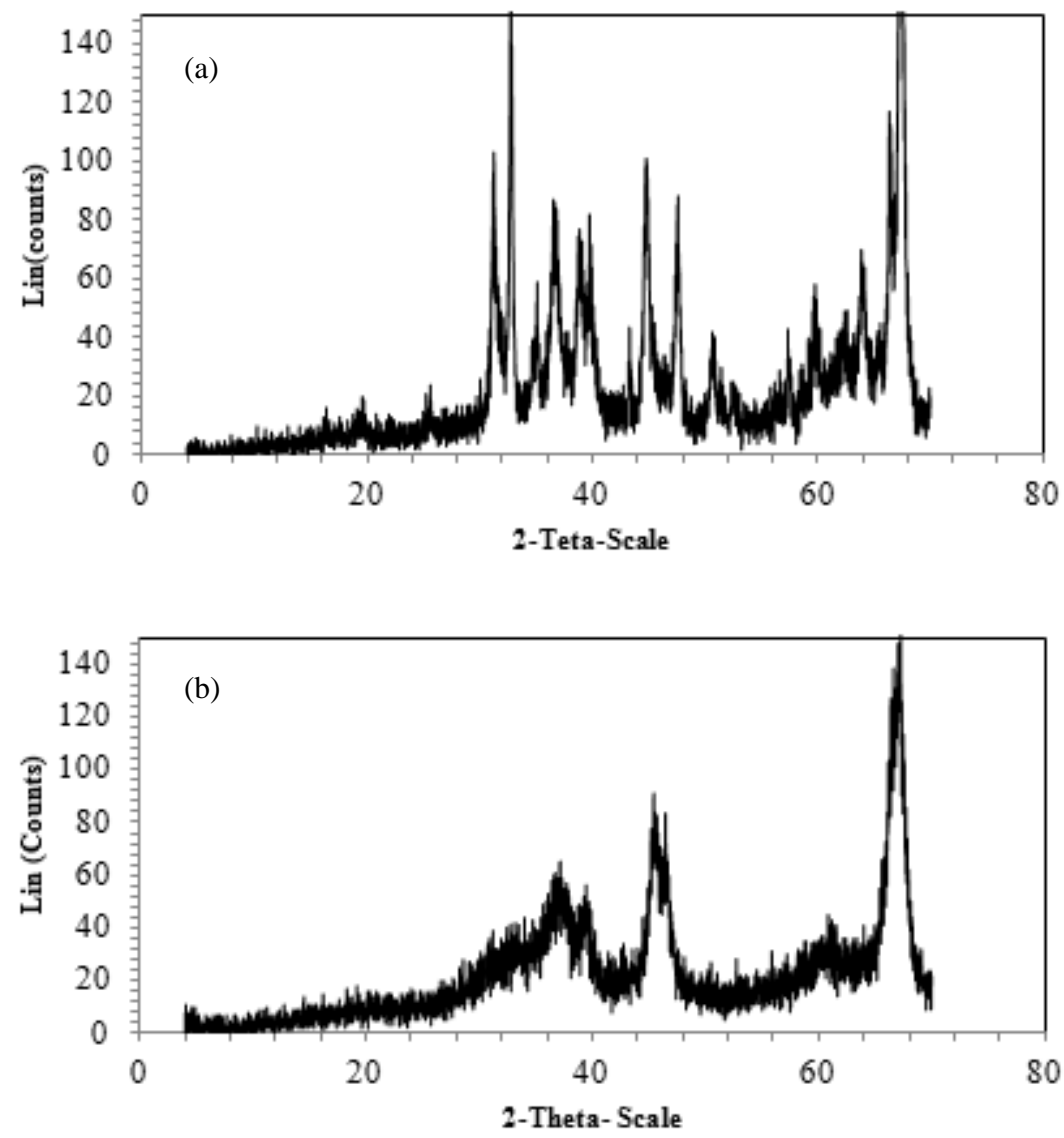

Fig 1 XRD results of (a) $\theta$-alumina, (b) $\gamma$-alumina

The TEM photos clearly show the size of platinum particles dispersed on the theta and gamma alumina. As shown in Fig. 2, large particles were observed on the $\mathrm{Pt} / \gamma-\mathrm{Al}_{2} \mathrm{O}_{3}$ catalyst. The size distribution of the platinum particles was wide in the range of 5-20 nm. On the other hand, the platinum particles dispersed on the $\mathrm{Pt} / \theta-\mathrm{Al}_{2} \mathrm{O}_{3}$ catalysts were very small and uniform. The histogram showed that their diameters were in the range of 1-3 nm. The most probable particle size of the $\mathrm{Pt} / \theta-\mathrm{Al}_{2} \mathrm{O}_{3}$ catalyst was $2 \mathrm{~nm}$, indicating an exceptionally high dispersion of platinum. 
Thus, it has been proved that phase conversion to theta is very effective in improving the dispersion of platinum impregnated on the alumina.

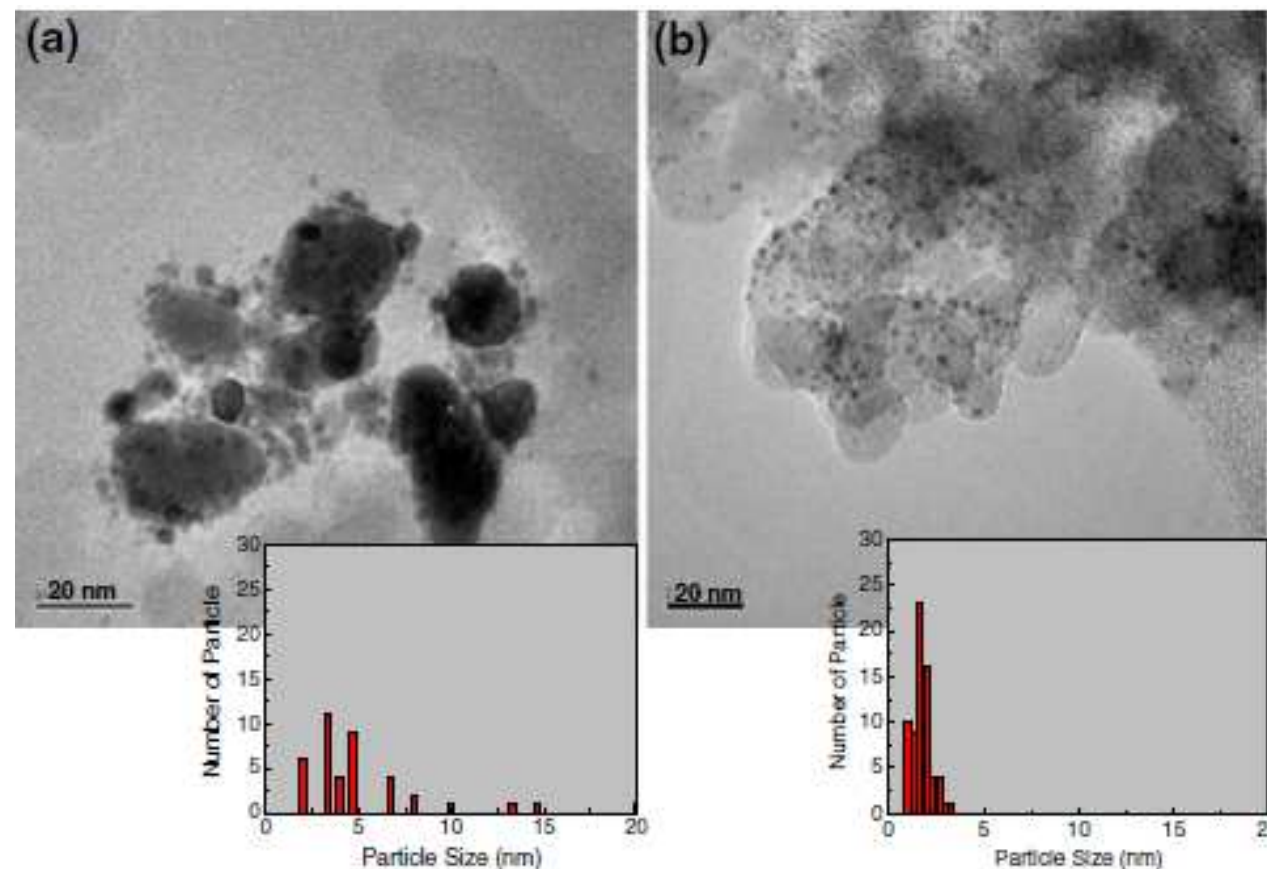

Fig 2 TEM photos of (a) Pt-Sn $/ \gamma-\mathrm{Al}_{2} \mathrm{O}_{3}$, (b) Pt-Sn/ $\theta-\mathrm{Al}_{2} \mathrm{O}_{3}$

The activities of platinum catalysts in dehydrogenation reaction are generally dependent on the dispersion of platinum because the platinum atoms dispersed on the surface work as active sites.

Activities of the Pt/ $/-\mathrm{Al}_{2} \mathrm{O}_{3}$ catalysts were higher than that of the $\mathrm{Pt} / \gamma-\mathrm{Al}_{2} \mathrm{O}_{3}$ catalyst in the dehydrogenation of propane. Performance of these catalysts is shown in Fig. 3. 

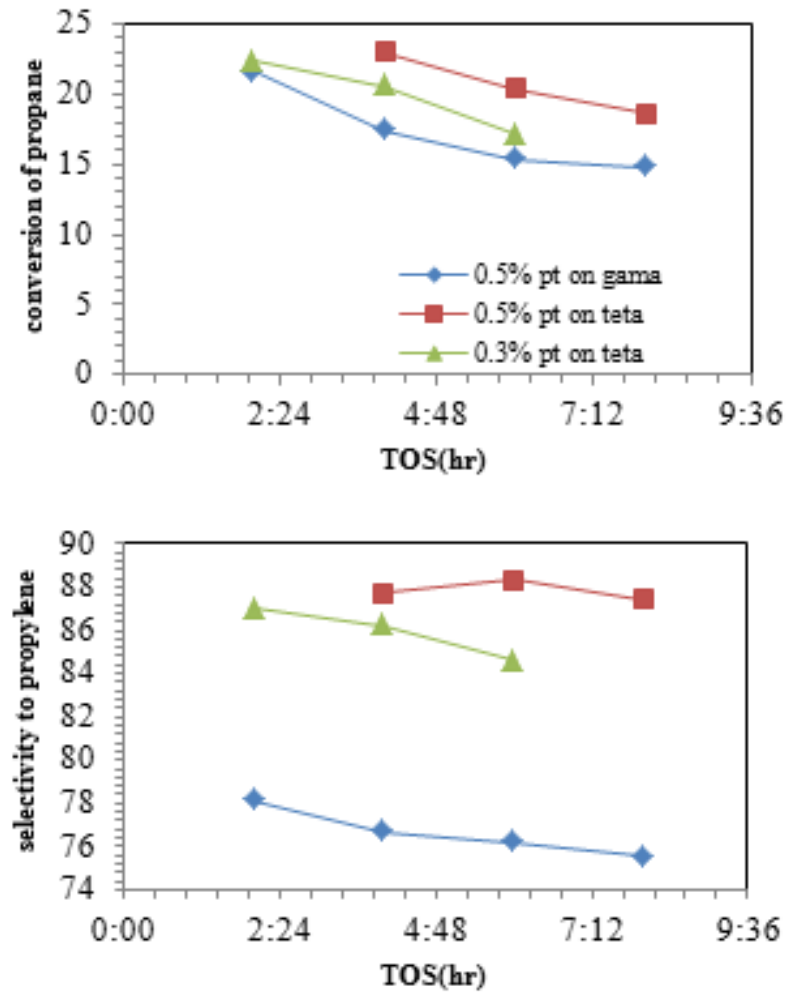

Fig 3 propane conversion and selectivity to propylene over catalysts, $\mathrm{T}=620^{\circ} \mathrm{C}, \mathrm{P}=0.86 \mathrm{bar}, \mathrm{H}_{2} / \mathrm{HC}=0.6, \mathrm{WHSV}=2.2$ $\mathrm{h}^{-1}$

As it can be seen from Fig. 4, even catalyst contains $0.3 \%$ platinum on $\theta$-alumina shows prior performance compared to catalyst contains $0.5 \%$ platinum on $\gamma$-alumina. Furthermore, catalysts which prepared on $\theta$-alumina show high resistance to poisoning by carbon deposition during propane dehydrogenation. This resistance is attributed to both the low acid sites of support and the small particle sizes which inhibit coke formation ${ }^{6}$. 


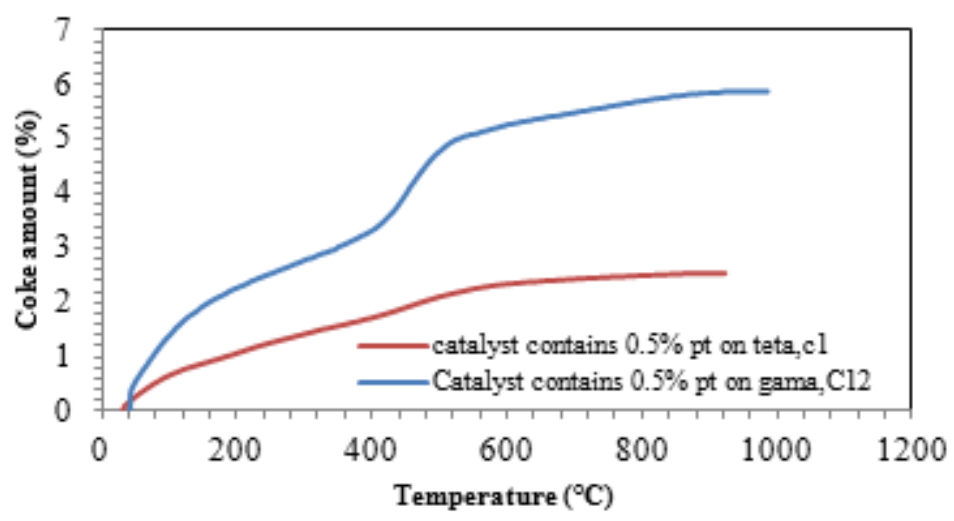

Fig 4 coke amount on catalysts

\section{Discussions}

The gamma phase converting to theta was effective in enhancing the dispersion of platinum. The TEM photos show a clear difference in the sizes of platinum particles in accordance with the methods of preparation employed. The platinum catalysts that were impregnated on the $\theta$ alumina support were more active in the dehydrogenation of propane. Moreover, the amounts of carbon monoxide adsorbed on platinum catalysts supported on $\theta$-alumina was far less than that of supported on $\gamma$-alumina. Different behavior of these catalysts can be attributed to both platinum dispersion and amount of acid sites. Additionally, strong metal-support interaction (SMSI) in $\gamma$-alumina leads to lower catalyst performance compared to those supported on $\theta$ alumina.

\section{Conclusions}

To the best of our knowledge, the theta phase has less acid sites than gamma, results in higher catalyst performance in dehydrogenation reaction and inhibiting coke formation on the catalyst. The size of the platinum particles dispersed on the catalysts prepared on $\theta$-support was uniform and in the range of $1-2 \mathrm{~nm}$, and the loading amount of platinum was in the range of $0.3-0.5 \mathrm{wt} . \%$. The activity of platinum catalyst in dehydrogenation reaction is generally dependent on the dispersion of platinum, let us to conclude that $\theta$-alumina is an appropriate support in dehydrogenation reaction.

\section{Acknowledgment}

The authors acknowledge NPC (R\&T) for their financial support of this study. The authors express their sincere gratitude to those who contributed to this research. 


\section{References}

\section{Journal paper}

1. Singh UK, Vannice MA (2001) Appl Catal A 213:1

2. Krishnankutty N, Vannice MA (1995) J Catal 155:312

3. Socolova NP (2004) Colloids Surf A 239:125

4. G.C. Bond, Heterogeneous Catalysis: Principles and Applications (Oxford University Press, 1987).

5. G.A. Somorjai, Catal. Lett. 9 (1991) 311.

6. Zou,W., and Gonzalez, R. D., J. Catal. 152, 291 (1995). 